Bull. Austral. Math. Soc.

$47 \mathrm{~A} 15,47 \mathrm{~B} 40$

VOL. $61(2000) \quad[11-26]$

\title{
AN INVARIANT SUBSPACE THEOREM ON SUBDECOMPOSABLE OPERATORS
}

\section{MingXue LiU}

H. Mohebi and M. Radjabalipour raised a conjecture on the invariant subspace problem in 1994. In this paper, we prove the conjecture under an additional condition, and obtain an invariant subspace theorem on subdecomposable operators.

In [9] Mohebi and Radjabalipour raised the following conjecture.

The Mohebi-Radjabalipour Conjecture. (See [9, p.236].) Assume the operators $T \in B(X)$ and $B \in B(Z)$ on Banach spaces $X$ and $Z$, and the nonempty open set $G$ in the complex plane $C$, satisfy the following conditions:

(1) $q T=B q$ for some injective $q \in B(X, Z)$ with a closed range $q X$.

(2) There exist sequences $\{G(n)\}$ of open sets and $\{M(n)\}$ of invariant subspaces of $B$ such that $\bar{G}(n) \subset G(n+1), G=\bigcup_{n} G(n), \sigma(B \mid M(n)) \subset$ $C \backslash G(n)$ and $\sigma(B / M(n)) \subset \bar{G}(n), n=1,2, \ldots$

(3) $\sigma(T)$ is dominating in $G$.

Then $T$ has a (non-trivial) invariant subspace.

It is easy to see that the Mohebi-Radjabalipour Conjecture, if true, will contain the main results of $[1,2,4,5,7,8,9]$ (and others) as special cases.

In the present article, using the $\mathrm{S}$. Brown Technique, we prove the MohebiRadjabalipour Conjecture under an additional condition. But the additional condition will be used in only one place, namely in the proof of Lemma 4 . Our main result is as follows.

THEOREM 1. Assume the operators $T \in B(X)$ and $B \in B(Z)$ on Banach spaces $X$ and $Z$, and the nonempty open set $G$ in $C$, satisfy conditions (1), (2) and (3) in the Mohebi-Radjabalipour Conjecture and the following additional condition:

(4) $\{q X+M(n)\}$ is a sequence of closed sets in $Z$.

Received 1st March, 1999

The author wishes to thank Professors Jiang Chunlan, Li Bingren, Ma Jipu, Sun Shanli, Wang Zongyao, Yan Zikun and Zhong Huaijie, and Doctors Guo Kunyu, Ji Youqing, Xu Shengzhi and other persons for their helpful discussions and suggestions during the 6th (China) National Conference on Functional Analysis.

The research was supported by the Open Foundation of (China) State Key Laboratory of Oil/gas Resevoir Geology and Exploitation, the Foundation of the Education Committee of Fujian Province and the Natural Science Foundation of Fujian Province of People's Republic of China.

Copyright Clearance Centre, Inc. Serial-fee code: 0004-9727/00 \$A2.00+0.00. 
Then $T$ has an invariant subspace.

To prove Theorem 1 we first recall some basic notation and facts, and give some lemmas.

We denote by $H^{\infty}(G)$ the Banach algebra of all bounded analytic functions on $G$ equipped with the norm $\|f\|=\sup \{|f(\lambda)| ; \lambda \in G\}$. It is well known that $H^{\infty}(G)$ is a $w^{*}$-closed subspace of $L^{\infty}(G)$ relative to the duality $\left\langle L^{1}(G), L^{\infty}(G)\right\rangle$ and that a sequence $\left\{f_{k}\right\}$ in $H^{\infty}(G)$ converges to zero relative to the $w^{*}$-topology if and only if it is norm-bounded and converges to zero uniformly on each compact subset of $G$. In particular, we can identify $H^{\infty}(G)$ with the dual space of the Banach space $Q=$ $L^{1}(G) /{ }^{\perp} H^{\infty}(G)$. Since $Q$ is separable, the above characterisation of $w^{*}$-convergent sequences in $H^{\infty}(G)$ immediately implies the $w^{*}$-continuity of all point evaluations $E_{\lambda}: H^{\infty}(G) \rightarrow C, f \rightarrow f(\lambda)(\lambda \in G)$.

For $f \in H^{\infty}(G)$ and $\lambda \in G$ we deonte by $f_{\lambda}$ the unique function in $H^{\infty}(G)$ with $(\lambda-\mu) f_{\lambda}(\mu)=f(\lambda)-f(\mu)$ for $\mu \in G$. It is easy to check that for fixed $\lambda \in G$ the map $H^{\infty}(G) \rightarrow H^{\infty}(G), f \rightarrow f_{\lambda}$, is $w^{*}$-continuous.

A subset $F$ of $C$ will be called dominating in $G$ if $\|f\|=\sup \{|f(\lambda)| ; \lambda \in F \cap G\}$ holds for all $f \in H^{\infty}(G)$.

Let $E$ be a Banach space. Then we denote the dual space of $E$ by $E^{*}$. If $M$ and $N$ are closed linear subspaces of $E$, then we set

$$
\alpha(M, N)=\inf \{\|x-y\| ; x \in M \text { with }\|x\|=1 \text { and } y \in N\}
$$

For $M \subset E$ arbitrary, by definition $\bigvee M$ denotes the closed linear hull of $M$. For $A \in B(E)$ the left essential spectrum $\sigma_{l e}(A)$ of $A$ is the set of those points $\lambda$ in $C$ such that $\operatorname{ran}(\lambda-A)$ is not closed or $\operatorname{dim} \operatorname{ker}(\lambda-A)=\infty$. For $A \in B(E)$, if $M$ is a closed linear subspace of $E$ with $A M \subset M$, then $A \mid M$ denotes the restriction of $A$ onto $M$, and $A / M$ denotes the quotient operator induced by $A$ on $E / M$. If $E$ and $F$ are Banach spaces, and $A \in B(E, F)$, then $A^{*}$ denotes the adjoint operator of $A$.

Throughout the rest of the present article, we shall assume that $X, Z, T, B, q, G$, $G(n)$ and $M(n)$ are as in Theorem 1.

Lemma 1. If $\sigma_{l e}\left(T^{*}\right) \neq \sigma\left(T^{*}\right)$, then $T$ has an invariant subspace.

Proof: The proof of Lemma 1 is routine, and is therefore omitted.

Note 1. By Lemma 1 and the condition (3) in Theorem 1, the proof of Theorem 1 can be reduced to the case in which $T$ satisfies the following additional condition:

$(3)^{\prime} \sigma_{l e}\left(T^{*}\right)$ is dominating in $G$.

By conditions (1) and (2) in Theorem 1, we can readily obtain Lemma 2 below. Moreover, Lemma 2 can be found in $[9$, Note 2$]$. 
LEMMA 2 .

(1) $q^{*} B^{*}=T^{*} q^{*}$ and $q^{*} \in B\left(Z^{*}, X^{*}\right)$ is surjective.

(2) For every natural number $n, M(n)^{\perp}$ is an invariant subspace of $B^{*}$ and

$$
\sigma\left(B^{*} \mid M(n)^{\perp}\right) \subset \bar{G}(n), \sigma\left(B^{*} / M(n)^{\perp}\right) \subset C \backslash G(n) .
$$

Lemma 3. Define $\widetilde{B}: q X \rightarrow q X$ by $\widetilde{B} \tilde{z}=B \tilde{z}$, and define $\widetilde{q}: X \rightarrow q X$ by $\widetilde{q} x=q x$. Then $\widetilde{B} \in B(q X), \widetilde{q} \in B(X, q X)$ and

(1) For any polynomial $p$, for any vectors $\widetilde{z} \in q X$ and $z^{*} \in Z^{*}$, we have

$$
\left\langle\widetilde{z}, p\left(\tilde{B}^{*}\right) \tilde{z^{*}}\right\rangle=\left\langle\widetilde{z}, p\left(B^{*}\right) z^{*}\right\rangle
$$

where $\tilde{z^{*}}$ denotes the restriction of $z^{*}$ onto $q X$, that is, $\tilde{z^{*}}=z^{*} \mid q X$.

(2) For any $x \in X$ and $z^{*} \in Z^{*}$, we have $\left\langle x, \widetilde{q}^{*} \widetilde{z^{*}}\right\rangle=\left\langle x, q^{*} z^{*}\right\rangle$ and $\left\|\tilde{q}^{*} \tilde{z}^{*}\right\|=$ $\left\|q^{*} z^{*}\right\|$, where $\tilde{z^{*}}=z^{*} \mid q X$.

Proof: Since $q T=B q$, it follows that $B q X \subset q X$. Hence $\widetilde{B}$ is a well-defined bounded linear operator on the Banach space $q X$, that is, $\widetilde{B} \in B(q X)$.

(1) Since $B q X \subset q X$, it follows that $p(B) q X \subset q X$. Consequently

$$
\left\langle\tilde{z}, p\left(B^{*}\right) z^{*}\right\rangle=\left\langle p(B) \tilde{z}, z^{*}\right\rangle=\left\langle p(B) \tilde{z}, \tilde{z}^{*}\right\rangle=\left\langle\tilde{z}, p\left(\widetilde{B}^{*}\right) \widetilde{z^{*}}\right\rangle .
$$

(2) It is clear that $\tilde{q} \in B(X, q X)$ and

$$
\left\langle x, \tilde{q}^{*} \tilde{z^{*}}\right\rangle=\left\langle q x, \tilde{z^{*}}\right\rangle=\left\langle q x, z^{*}\right\rangle=\left\langle x, q^{*} z^{*}\right\rangle \text {. }
$$

Therefore $\left\|\tilde{q}^{*} \tilde{z^{*}}\right\|=\left\|q^{*} z^{*}\right\|$.

LEMMA 4. For each natural number $n$, set

$$
M(n)^{\perp} \mid q X=\left\{\widetilde{z^{*}} \in(q X)^{*} ; \text { there is } z^{*} \in M(n)^{\perp} \text { with } z^{*} \mid q X=\tilde{z^{*}}\right\}
$$

Then $M(n)^{\perp} \mid q X$ is a closed linear subspace in the Banach space $(q X)^{*}$.

Proof: It is obvious that $M(n)^{\perp} \mid q X$ is a linear subspace in $(q X)^{*}$. To show that $M(n)^{\perp} \mid q X$ is closed in $(q X)^{*}$, it suffices to show that for any sequence $\left\{\widetilde{z_{m}^{*}}\right\}$ in $M(n)^{\perp} \mid q X$, if

$$
\lim _{m \rightarrow \infty} \widetilde{z_{m}^{*}}=\widetilde{z_{0}^{*}} \in(q X)^{*}
$$


then $\tilde{z_{0}^{*}} \in M(n)^{\perp} \mid q X$.

In fact, since $\widetilde{z_{m}^{*}} \in M(n)^{\perp} \mid q X$, there exists $z_{m}^{*} \in M(n)^{\perp}$ such that $\widetilde{z_{m}^{*}}=$ $z_{m}^{*} \mid q X$. Hence, it follows from (4.1) that

$$
\lim _{m \rightarrow \infty}\left\langle z, z_{m}^{*}\right\rangle=\left\langle z, \tilde{z_{0}^{*}}\right\rangle
$$

for any $z \in q X$. Since $z_{m}^{*} \in M(n)^{\perp}$, it follows that $\left\langle z, z_{m}^{*}\right\rangle=0$ for any $z \in M(n)$. Therefore, for any $z \in M(n)$ we have

$$
\lim _{m \rightarrow \infty}\left\langle z, z_{m}^{*}\right\rangle=0
$$

Since $z_{m}^{*} \in Z^{*}, z_{m}^{*}$ is well-defined on $q X+M(n)$. Thereby, it follows from (4.2) and (4.3) that for any $z \in q X+M(n)$ the sequence $\left\{\left\langle z, z_{m}^{*}\right\rangle\right\}$ of numbers is convergent. Set

$$
\lim _{m \rightarrow \infty}\left\langle z, z_{m}^{*}\right\rangle=\varphi(z), \quad z \in q X+M(n)
$$

Define the functional $z_{0}^{* \prime}$ acting on $q X+M(n)$ by the equation

$$
\left\langle z, z_{0}^{* \prime}\right\rangle=\varphi(z), \quad z \in q X+M(n) .
$$

Obviously, $z_{0}^{* \prime}$ is a linear functional and

$$
\left\langle z, z_{0}^{* \prime}\right\rangle= \begin{cases}\left\langle z, \tilde{z_{0}^{*}}\right\rangle, & z \in q X, \\ 0, & z \in M(n) .\end{cases}
$$

Since $q X, M(n)$ and $q X+M(n)$ are closed in the Banach space $Z$, by the Open Mapping Theorem there exists a real number $K>0$ such that for any $z \in q X+$ $M(n)$ there are vectors $z_{1} \in q X, z_{2} \in M(n)$ with $z=z_{1}+z_{2}$ and $\left\|z_{1}\right\| \leqslant K\|z\|$. Consequently, we have

$$
\left|\left\langle z, z_{0}^{* \prime}\right\rangle\right|=\left|\left\langle z_{1}, \tilde{z_{0}^{*}}\right\rangle\right| \leqslant\left\|\tilde{z_{0}^{*}}\right\|\left\|z_{1}\right\| \leqslant K\left\|\tilde{z_{0}^{*}}\right\|\|z\| .
$$

Thus $z_{0}^{* \prime}$ is a bounded linear functional on $q X+M(n)$. By applying the Hahn-Banach Theorem we can find $z_{0}^{*} \in Z^{*}$ such that $z_{0}^{*} \mid(q X+M(n))=z_{0}^{* \prime}$. Thus by (4.4) we get $z_{0}^{*} \in M(n)^{\perp}$ and $\widetilde{z_{0}^{*}}=z_{0}^{*}\left|q X \in M(n)^{\perp}\right| q X$.

LEMMA 5. Let $n$ be a natural number. If $\lambda \in G(n)$, then $\lambda-\widetilde{B}^{*} /\left(M(n)^{\perp} \mid q X\right)$ is a bijective bounded linear operator from $(q X)^{*} /\left(M(n)^{\perp} \mid q X\right)$ onto $(q X)^{*} /\left(M(n)^{\perp} \mid q X\right)$.

Proof: Since $M(n)^{\perp}$ is an invariant subspace of $B^{*}$, it follows from Lemmas 3 and 4 that $M(n)^{\perp} \mid q X$ is a closed linear subspace in $(q X)^{*}$ and $\widetilde{B}^{*}\left(M(n)^{\perp} \mid(q X)\right)$ 
$\subset M(n)^{\perp} \mid q X$. Therefore, $\lambda-\widetilde{B}^{*} /\left(M(n)^{\perp} \mid q X\right)$ is a bounded linear operator from $(q X)^{*} /\left(M(n)^{\perp} \mid q X\right)$ into $(q X)^{*} /\left(M(n)^{\perp} \mid q X\right)$.

By applying Lemma 2, we get $\lambda \in \rho\left(B^{*} / M(n)^{\perp}\right)$. Consequently, $\lambda-B^{*} / M(n)^{\perp}$ is a bijective bounded linear operator from $Z^{*} / M(n)^{\perp}$ onto $Z^{*} / M(n)^{\perp}$.

First we show that $\lambda-\widetilde{B}^{*} /\left(M(n)^{\perp} \mid q X\right)$ is surjective from $(q X)^{*} /\left(M(n)^{\perp} \mid q X\right)$ onto $(q X)^{*} /\left(M(n)^{\perp} \mid q X\right)$. In fact, for any $\tilde{z}_{0}^{*}+M(n)^{\perp} \mid q X \in(q X)^{*} /\left(M(n)^{\perp} \mid q X\right)$, by the Hahn-Banach Theorem there exists $z_{0}^{*} \in Z^{*}$ such that $\tilde{z_{0}^{*}}=z_{0}^{*} \mid q X$. Since $\lambda-B^{*} / M(n)^{\perp}$ is surjective from $Z^{*} / M(n)^{\perp}$ onto $Z^{*} / M(n)^{\perp}$, there is a vector $z^{*}+$ $M(n)^{\perp} \in Z^{*} / M(n)^{\perp}$ such that

$$
\left(\lambda-B^{*}\right) z^{*}+M(n)^{\perp}=\left(\lambda-B^{*} / M(n)^{\perp}\right)\left(z^{*}+M(n)^{\perp}\right)=z_{0}^{*}+M(n)^{\perp} .
$$

Hence

$$
\left(\left(\lambda-B^{*}\right) z^{*}\right)\left|q X+M(n)^{\perp}\right| q X=\tilde{z_{0}^{*}}+M(n)^{\perp} \mid q X .
$$

Set $\tilde{z^{*}}=z^{*} \mid q X$. Then it follows from Lemma 3 and (5.1) that

$$
\begin{aligned}
\left(\lambda-\widetilde{B}^{*} /\left(M(n)^{\perp} \mid q X\right)\right)\left(\tilde{z^{*}}+M(n)^{\perp} \mid q X\right) & =\left(\lambda-\widetilde{B}^{*}\right) \tilde{z}^{*}+M(n)^{\perp} \mid q X \\
& =\left(\left(\lambda-B^{*}\right) z^{*}\right)\left|q X+M(n)^{\perp}\right| q X \\
& =\tilde{z_{0}^{*}}+M(n)^{\perp} \mid q X .
\end{aligned}
$$

Next it will be shown that $\lambda-\widetilde{B}^{*} /\left(M(n)^{\perp} \mid q X\right)$ is injective from $(q X)^{*} /\left(M(n)^{\perp} \mid q X\right)$ onto $(q X)^{*} /\left(M(n)^{\perp} \mid q X\right)$. It is sufficient to show that for any $\tilde{z^{*}}+M(n)^{\perp} \mid q X \in$ $(q X)^{*} /\left(M(n)^{\perp} \mid q X\right)$, if $\left(\lambda-\widetilde{B}^{*} /\left(M(n)^{\perp} \mid q X\right)\right)\left(\tilde{z^{*}}+M(n)^{\perp} \mid q X\right)=0$, then $\left(\tilde{z^{*}}+\right.$ $\left.M(n)^{\perp} \mid q X\right)=0$. That is, it is sufficient to show that for any $\tilde{z}^{*}+M(n)^{\perp} \mid q X \in$ $(q X)^{*} /\left(M(n)^{\perp} \mid q X\right)$, if

$$
\left(\lambda-\tilde{B}^{*}\right) \tilde{z^{*}}+M(n)^{\perp} \mid q X=0
$$

then $\tilde{z^{*}}+M(n)^{\perp} \mid q X=0$.

In fact, since $\lambda-B^{*} / M(n)^{\perp}$ is surjective from $Z^{*} / M(n)^{\perp}$ onto $Z^{*} / M(n)^{\perp}$, it follows that

$$
\begin{aligned}
Z^{*} / M(n)^{\perp} & =\left\{\left(\lambda-B^{*} / M(n)^{\perp}\right)\left(z^{*}+M(n)^{\perp}\right) ; z^{*}+M(n)^{\perp} \in Z^{*} / M(n)^{\perp}\right\} \\
& =\left\{\left(\lambda-B^{*}\right) z^{*}+M(n)^{\perp} ; z^{*} \in Z^{*}\right\} .
\end{aligned}
$$

Similarly, since $\lambda-\widetilde{B}^{*} /\left(M(n)^{\perp} \mid q X\right)$ is surjective from $(q X)^{*} /\left(M(n)^{\perp} \mid q X\right)$ onto $(q X)^{*} /\left(M(n)^{\perp} \mid q X\right)$, it follows that

$$
(q X)^{*} /\left(M(n)^{\perp} \mid q X\right)=\left\{\left(\lambda-\widetilde{B}^{*}\right) \tilde{z}^{*}+M(n)^{\perp} \mid q X ; \tilde{z^{*}} \in(q X)^{*}\right\} .
$$


Therefore we can define a map $A: Z^{*} / M(n)^{\perp} \rightarrow(q X)^{*} /\left(M(n)^{\perp} \mid q X\right)$ by

$$
A\left(\left(\lambda-B^{*}\right) z^{*}+M(n)^{\perp}\right)=\left(\lambda-\widetilde{B}^{*}\right) \tilde{z^{*}}+M(n)^{\perp} \mid q X
$$

where $\tilde{z^{*}}=z^{*} \mid q X$. It is easy to see that the image point of the vector $\left(\lambda-B^{*}\right) z^{*}+$ $M(n)^{\perp} \in Z^{*} / M(n)^{\perp}$ under $A$ is independent of the particular choice of $\left(\lambda-B^{*}\right) z^{*}$, that is, if $\left(\lambda-B^{*}\right) z_{1}^{*}+M(n)^{\perp}=\left(\lambda-B^{*}\right) z_{2}^{*}+M(n)^{\perp}$ in the quotient space $Z^{*} / M(n)^{\perp}$, then

$$
A\left(\left(\lambda-B^{*}\right) z_{1}^{*}+M(n)^{\perp}\right)=A\left(\left(\lambda-B^{*}\right) z_{2}^{*}+M(n)^{\perp}\right) .
$$

So $A$ is well defined.

It is plain that $A$ is a linear operator. We now show that $A$ is bounded. Indeed, for any $z^{* \prime} \in M(n)^{\perp}$, set $\widetilde{z^{* \prime}}=z^{* \prime} \mid q X$, then $\widetilde{z^{* \prime}} \in M(n)^{\perp} \mid q X$. Thus by Lemma 3 we obtain

$$
\left\|\left(\lambda-\widetilde{B}^{*}\right) \tilde{z^{*}}+\widetilde{z^{* \prime}}\right\|=\left\|\left(\left(\lambda-B^{*}\right) z^{*}+z^{* \prime}\right) \mid q X\right\| \leqslant\left\|\left(\lambda-B^{*}\right) z^{*}+z^{* \prime}\right\|
$$

for every $z^{*} \in Z^{*}$, where $\tilde{z^{*}}=z^{*} \mid q X$. Consequently

$$
\begin{aligned}
\left\|A\left(\left(\lambda-B^{*}\right) z^{*}+M(n)^{\perp}\right)\right\| & =\left\|\left(\lambda-\widetilde{B}^{*}\right) \tilde{z^{*}}+M(n)^{\perp} \mid q X\right\| \\
& =\inf \left\{\left\|\left(\lambda-\widetilde{B^{*}}\right) \tilde{z^{*}}+\widetilde{z^{* \prime}}\right\| ; \widetilde{z^{* \prime}} \in M(n)^{\perp} \mid q X\right\} \\
& \leqslant \inf \left\{\left\|\left(\lambda-B^{*}\right) z^{*}+z^{* \prime}\right\| ; z^{* \prime} \in M(n)^{\perp}\right\} \\
& =\left\|\left(\lambda-B^{*}\right) z^{*}+M(n)^{\perp}\right\| .
\end{aligned}
$$

It is obvious that $A$ is surjective from the Banach space $Z^{*} / M(n)^{\perp}$ onto the Banach space $(q X)^{*} /\left(M(n)^{\perp} \mid q X\right)$. Thus the Open Mapping Theorem implies there exists a real number $K>0$ such that for any

$$
\left(\lambda-\widetilde{B}^{*}\right) \widetilde{z^{*}}+M(n)^{\perp} \mid q X \in(q X)^{*} /\left(M(n)^{\perp} \mid q X\right)
$$

there is a vector $\left(\lambda-B^{*}\right) z^{*}+M(n)^{\perp} \in Z^{*} / M(n)^{\perp}$ with

$$
A\left(\left(\lambda-B^{*}\right) z^{*}+M(n)^{\perp}\right)=\left(\lambda-\widetilde{B}^{*}\right) \tilde{z^{*}}+M(n)^{\perp} \mid q X
$$

and

$$
\left\|\left(\lambda-B^{*}\right) z^{*}+M(n)^{\perp}\right\| \leqslant K\left\|\left(\lambda-\widetilde{B}^{*}\right) \tilde{z}^{*}+M(n)^{\perp} \mid q X\right\| .
$$


Therefore, if $\left(\lambda-\widetilde{B}^{*}\right) \widetilde{z^{*}}+M(n)^{\perp} \mid q X=0$, then

$$
\left(\lambda-B^{*} / M(n)^{\perp}\right)\left(z^{*}+M(n)^{\perp}\right)=\left(\lambda-B^{*}\right) z^{*}+M(n)^{\perp}=0 .
$$

Since $\lambda-B^{*} / M(n)^{\perp}$ is injective from $Z^{*} / M(n)^{\perp}$ onto $Z^{*} / M(n)^{\perp}$, it follows from (5.3) that $z^{*}+M(n)^{\perp}=0$. Hence $z^{*} \in M(n)^{\perp}$. Thus by (5.2) we get

$$
\tilde{z^{*}}=z^{*}\left|q X \in M(n)^{\perp}\right| q X .
$$

Therefore $\tilde{z^{*}}+M(n)^{\perp} \mid q X=0$.

Lemma 6. Let $n$ be a natural number. If $\lambda \in \sigma_{l e}\left(T^{*}\right) \cap G(n)$, then for each closed finite codimensional subspace $X_{0}^{*}$ in $X^{*}$, there exist sequences $\left\{x_{m}^{*}\right\}$ in $X_{0}^{*}$, $\left\{z_{m}^{*}\right\}$ in $Z^{*}$ and $\left\{\widetilde{z_{m}^{* \prime}}\right\}$ in $M(n)^{\perp} \mid q X$ such that

$$
\left\|x_{m}^{*}\right\|=1, \widetilde{q^{*}} \widetilde{z_{m}^{*}}=x_{m}^{*}, \lim _{m \rightarrow \infty}\left(\lambda-\widetilde{B}^{*}\right) \widetilde{z_{m}^{*}}=0, \lim _{m \rightarrow \infty}\left(\widetilde{z_{m}^{*}}-\widetilde{z_{m}^{* \prime}}\right)=0,
$$

where $\widetilde{z_{m}^{*}}=z_{m}^{*} \mid q X$.

Proof: By $[7$, Lemma 0.1$]$, there exists a sequence $\left\{x_{m}^{*}\right\}$ in $X_{0}^{*}$ such that $\left\|x_{m}^{*}\right\|=$ 1 and

$$
\lim _{m \rightarrow \infty}\left(\lambda-T^{*}\right) x_{m}^{*}=0 .
$$

Therefore by Lemma 2 we can find a sequence $\left\{z_{m}^{*}\right\}$ in $Z^{*}$ such that $x_{m}^{*}=q^{*} z_{m}^{*}$ for $m=1,2, \ldots$, and

$$
\lim _{m \rightarrow \infty} q^{*}\left(\lambda-B^{*}\right) z_{m}^{*}=\lim _{m \rightarrow \infty}\left(\lambda-T^{*}\right) q^{*} z_{m}^{*}=\lim _{m \rightarrow \infty}\left(\lambda-T^{*}\right) x_{m}^{*}=0 .
$$

Thus by Lemma 3 we obtain $\widetilde{q^{*}} \widetilde{z_{m}^{*}}=x_{m}^{*}$ for $m=1,2, \ldots$, and

$$
\lim _{m \rightarrow \infty}\left\|\widetilde{q}^{*}\left(\lambda-\widetilde{B}^{*}\right) \widetilde{z_{m}^{*}}\right\|=\lim _{m \rightarrow \infty}\left\|q^{*}\left(\lambda-B^{*}\right) z_{m}^{*}\right\|=0
$$

where $\widetilde{z_{m}^{*}}=z_{m}^{*} \mid q X$.

Since $q \in B(X, Z)$ is an injection with a closed range $q X, \widetilde{q} \in B(X, q X)$ is bijective from the Banach space $X$ onto the Banach space $q X$. Thus by the Inverse Mapping Theorem and (6.1) we have

$$
\lim _{m \rightarrow \infty}\left(\lambda-\widetilde{B}^{*}\right) \widetilde{z_{m}^{*}}=\lim _{m \rightarrow \infty}\left(\widetilde{q}^{*}\right)^{-1} \widetilde{q}^{*}\left(\lambda-\widetilde{B}^{*}\right) \widetilde{z_{m}^{*}}=0 .
$$

Consequently,

$$
\begin{aligned}
\left\|\left(\lambda-\widetilde{B}^{*} /\left(M(n)^{\perp} \mid q X\right)\right)\left(\widetilde{z_{m}^{*}}+M(n)^{\perp} \mid q X\right)\right\| & =\left\|\left(\lambda-\widetilde{B}^{*}\right) \widetilde{z_{m}^{*}}+M(n)^{\perp} \mid q X\right\| \\
& \leqslant\left\|\left(\lambda-\widetilde{B}^{*}\right) \widetilde{z_{m}^{*}}\right\| \rightarrow 0, \text { as } m \rightarrow \infty .
\end{aligned}
$$


Thus by Lemma 5 we get

$$
\begin{aligned}
& \inf \left\{\left\|\widetilde{z_{m}^{*}}-\tilde{z^{*}}\right\| ; \tilde{z^{*}} \in M(n)^{\perp} \mid q X\right\}=\left\|\widetilde{z_{m}^{*}}+M(n)^{\perp} \mid q X\right\| \\
& =\left\|\left(\lambda-\widetilde{B}^{*} /\left(M(n)^{\perp} \mid q X\right)\right)^{-1}\left(\lambda-\widetilde{B}^{*} /\left(M(n)^{\perp} \mid q X\right)\right)\left(\widetilde{z_{m}^{*}}+M(n)^{\perp} \mid q X\right)\right\| \\
& \rightarrow 0 \text {, as } m \rightarrow \infty \text {. }
\end{aligned}
$$

Therefore, there is a sequence $\left\{\widetilde{z_{m}^{* \prime}}\right\}$ in $M(n)^{\perp} \mid q X$ such that $\lim _{m \rightarrow \infty}\left(\widetilde{z_{m}^{*}}-\widetilde{z_{m}^{* \prime}}\right)=0$.

Note 2. By Lemma 6 we can find a natural number $n_{0}$ such that $M(n)^{\perp} \mid q X \neq\{0\}$ for all $n \geqslant n_{0}$. In fact, by Note $1, \sigma_{l e}\left(T^{*}\right)$ is dominating in $G$. Thus by the condition (2) in Theorem 1, there is a natural number $n_{0}$ such that for each $n \geqslant n_{0}, \sigma_{l e}\left(T^{*}\right) \cap G(n)$ is nonempty. Thus by Lemma 6 , if $M(n)^{\perp} \mid q X=\{0\}$ for some $n \geqslant n_{0}$, then for any given closed finite codimensional subspace $X_{0}^{*}$ in $X^{*}$, there are sequences $\left\{x_{m}^{*}\right\}$ in $X_{0}^{*}$ and $\left\{z_{m}^{*}\right\}$ in $Z^{*}$ such that $\left\|\widetilde{q^{*}} \widetilde{z_{m}^{*}}\right\|=\left\|x_{m}^{*}\right\|=1$ and $\lim _{m \rightarrow \infty} \widetilde{z_{m}^{*}}=0$, where $\widetilde{z_{m}^{*}}=z_{m}^{*} \mid q X$. Therefore $\lim _{m \rightarrow \infty}\left\|\widetilde{q}^{*} \widetilde{z_{m}^{*}}\right\|=0$. This contradicts $\left\|\widetilde{q}^{*} \widetilde{z_{m}^{*}}\right\|=1$. Thus $M(n)^{\perp} \mid q X \neq\{0\}$ for all $n \geqslant n_{0}$.

From now on fix $n_{0}$.

LEMMA 7. Let $n$ be a natural number. If $\lambda \in C \backslash \bar{G}(n)$, then $\lambda-\widetilde{B}^{*} \mid\left(M(n)^{\perp} \mid q X\right)$ is a bijective bounded linear operator from $M(n)^{\perp} \mid q X$ onto $M(n)^{\perp} \mid q X$.

Proof: Since $M(n)^{\perp}$ is an invariant subspace of $B^{*}$, it follows from Lemmas 3 and 4 that $M(n)^{\perp} \mid q X$ is a closed linear subspace in $(q X)^{*}$ and $\widetilde{B}^{*}\left(M(n)^{\perp} \mid q X\right) \subset$ $M(n)^{\perp} \mid q X$. Therefore, $\lambda-\widetilde{B}^{*} \mid\left(M(n)^{\perp} \mid q X\right)$ is a bounded linear operator from $M(n)^{\perp} \mid q X$ into $M(n)^{\perp} \mid q X$.

By applying Lemma 2, we obtain $\lambda \in \rho\left(B^{*} \mid M(n)^{\perp}\right)$. Consequently, $\lambda-B^{*} \mid M(n)^{\perp}$ is a bijective bounded linear operator from $M(n)^{\perp}$ onto $M(n)^{\perp}$.

First we show that $\lambda-\widetilde{B}^{*} \mid\left(M(n)^{\perp} \mid q X\right)$ is surjective from $M(n)^{\perp} \mid q X$ onto $M(n)^{\perp} \mid q X$. In fact, for any $\tilde{z_{0}^{*}} \in M(n)^{\perp} \mid q X$, there exists $z_{0}^{*} \in M(n)^{\perp}$ such that $\widetilde{z_{0}^{*}}=z_{0}^{*} \mid q X$. Since $\lambda-B^{*} \mid M(n)^{\perp}$ is surjective from $M(n)^{\perp}$ onto $M(n)^{\perp}$, there is $z^{*} \in M(n)^{\perp}$ such that

$$
\left(\lambda-B^{*}\right) z^{*}=\left(\lambda-B^{*} \mid M(n)^{\perp}\right) z^{*}=z_{0}^{*}
$$

Set $\widetilde{z^{*}}=z^{*} \mid q X$, then $\widetilde{z^{*}} \in M(n)^{\perp} \mid q X$. Thus by Lemma 3 and (7.1) we have

$$
\left(\lambda-\widetilde{B}^{*}\right) \widetilde{z^{*}}=\left(\left(\lambda-B^{*}\right) z^{*}\right) \mid q X=\tilde{z_{0}^{*}}
$$


Next it will be shown that $\lambda-\widetilde{B}^{*} \mid\left(M(n)^{\perp} \mid q X\right)$ is injective from $M(n)^{\perp} \mid q X$ onto $M(n)^{\perp} \mid q X$. It will be sufficient to show that for any $\widetilde{z^{*}} \in M(n)^{\perp} \mid q X$, if $\left(\lambda-\widetilde{B}^{*}\right) \widetilde{z}^{*}=$ 0 , then $\tilde{z^{*}}=0$.

In fact, since $\lambda-B^{*} \mid M(n)^{\perp}$ is surjective from $M(n)^{\perp}$ onto $M(n)^{\perp}$, it is true that

$$
M(n)^{\perp}=\left\{\left(\lambda-B^{*}\right) z^{*} ; z^{*} \in M(n)^{\perp}\right\} .
$$

Similarly, since $\lambda-\widetilde{B}^{*} \mid\left(M(n)^{\perp} \mid q X\right)$ is surjective from $M(n)^{\perp} \mid q X$ onto $M(n)^{\perp} \mid q X$, it is true that

$$
M(n)^{\perp} \mid q X=\left\{\left(\lambda-\widetilde{B}^{*}\right) \widetilde{z^{*}} ; \widetilde{z^{*}} \in M(n)^{\perp} \mid q X\right\} .
$$

Therefore, we can define a map $S: M(n)^{\perp} \rightarrow M(n)^{\perp} \mid q X$ by

$$
S\left(\left(\lambda-B^{*}\right) z^{*}\right)=\left(\lambda-\tilde{B}^{*}\right) \tilde{z^{*}}
$$

where $\tilde{z^{*}}=z^{*} \mid q X$. It is easy to see that $S$ is well defined.

By Lemma 3, we get

$$
\left\|S\left(\lambda-B^{*}\right) z^{*}\right\|=\left\|\left(\lambda-\widetilde{B}^{*}\right) \tilde{z}^{*}\right\|=\left\|\left(\left(\lambda-B^{*}\right) z^{*}\right) \mid q X\right\| \leqslant\left\|\left(\lambda-B^{*}\right) z^{*}\right\| .
$$

Therefore, $S$ is a bounded linear operator. Moreover, for any $\left(\lambda-\widetilde{B}^{*}\right) \tilde{z}^{*} \in M(n)^{\perp} \mid q X$ with $\tilde{z^{*}} \in M(n)^{\perp} \mid q X$, there exists $z^{*} \in M(n)^{\perp}$ such that $\tilde{z^{*}}=z^{*} \mid q X$. Hence $S\left(\left(\lambda-B^{*}\right) z^{*}\right)=\left(\lambda-\widetilde{B}^{*}\right) \tilde{z^{*}}$. Consequently, $S$ is a surjective bounded linear operator from the Banach space $M(n)^{\perp}$ onto the Banach space $M(n)^{\perp} \mid q X$. Thus by the Open Mapping Theorem there exists a real number $K>0$ such that for any $\left(\lambda-\widetilde{B}^{*}\right) \tilde{z^{*}} \in M(n)^{\perp} \mid q X$ with $\tilde{z^{*}} \in M(n)^{\perp} \mid q X$, there is a vector $\left(\lambda-B^{*}\right) z^{*} \in M(n)^{\perp}$ with $z^{*} \in M(n)^{\perp}$,

$$
S\left(\left(\lambda-B^{*}\right) z^{*}\right)=\left(\lambda-\widetilde{B}^{*}\right) \widetilde{z^{*}}
$$

and

$$
\left\|\left(\lambda-B^{*}\right) z^{*}\right\| \leqslant K\left\|\left(\lambda-\widetilde{B}^{*}\right) \tilde{z^{*}}\right\| .
$$

Therefore, if $\left(\lambda-\widetilde{B}^{*}\right) \tilde{z^{*}}=0$, then $\left(\lambda-B^{*}\right) z^{*}=0$. Since $\lambda-B^{*} \mid M(n)^{\perp}$ is an injection from $M(n)^{\perp}$ onto $M(n)^{\perp}$. It follows that $z^{*}=0$. Thus by (7.2) we obtain

$$
\widetilde{z^{*}}=z^{*} \mid q X=0
$$


and this concludes the proof of Lemma 7.

To prove Thoerem 1, we need to construct some $w^{*}$-continuous linear functionals acting on $H^{\infty}(G)$. By Lemma 2 and [9, Lemma 1.2], we obtain $M(n)^{\perp} \subset M(n+1)^{\perp}$ for $n=1,2, \ldots$. Therefore $M(n)^{\perp}\left|q X \subset M(n+1)^{\perp}\right| q X$ for $n=1,2, \ldots$ Set

$$
M(G)=\bigcup_{n}\left(M(n)^{\perp} \mid q X\right) .
$$

For any $x \in X, \tilde{z^{*}} \in M(G)$, choose a natural number $n \geqslant n_{0}$ such that $\tilde{z^{*}} \epsilon$ $M(n)^{\perp} \mid q X$. By Note 2 and Lemma 7 , we have

$$
\sigma\left(\widetilde{B}^{*} \mid\left(M(n)^{\perp} \mid q X\right)\right) \subset \bar{G}(n) \subset G .
$$

Consequently, we can define a functional $x \otimes \widetilde{z^{*}}: H^{\infty}(G) \rightarrow C$ by

$$
x \otimes \tilde{z^{*}}(f)=\left\langle x, \widetilde{q}^{*} f\left(\widetilde{B}_{n}^{*}\right) \tilde{z^{*}}\right\rangle
$$

where $\widetilde{B}_{n}^{*}$ denotes $\widetilde{B}^{*} \mid\left(M(n)^{\perp} \mid q X\right)$, and $f\left(\widetilde{B}_{n}^{*}\right)$ is defined by the Riesz-Dunford functional calculus with analytic functions. As in [2], we can show that for any $x \in X$ and $\widetilde{z^{*}} \in M(G), x \otimes \widetilde{z^{*}}$ is a well-defined $w^{*}$-continuous linear functional which is independent of the particular choice of $n$.

Lemma 8. (Similar to [8, Proposition 2.8], or [4, Lemmas 4.2 and 4.3], et cetera.) Let $r, s$ be natural numbers. Consider non-negative real numbers $c_{1}, \ldots, c_{r}$ with $c_{1}+\cdots+c_{r}=1$ and complex numbers $\lambda_{1}, \ldots, \lambda_{r} \in \sigma_{l e}\left(T^{*}\right) \cap G$. If $a_{1}, \ldots, a_{s} \in$ $X, \tilde{b_{1}^{*}}, \ldots, \widetilde{b_{s}^{*}} \in M(G)$ and $\varepsilon>0$ are arbitrary, then there are vectors $x \in X, \widetilde{z^{*}} \in$ $M(G)$ such that $\|x\| \leqslant 3,\left\|\widetilde{q}^{*} \tilde{z}^{*}\right\| \leqslant 2$ and

$$
\begin{aligned}
& \text { (1) }\left\|x \otimes \widetilde{z^{*}}-\sum_{k=1}^{r} c_{k} E_{\lambda_{k}}\right\| \leqslant \varepsilon, \\
& \text { (2) }\left\|x \otimes \widetilde{b_{j}^{*}}\right\| \leqslant \varepsilon,\left\|a_{j} \otimes \widetilde{z^{*}}\right\| \leqslant \varepsilon, j=1,2, \ldots, s .
\end{aligned}
$$

Proof: We start by choosing an arbitrary real number $\delta$ with $0<\delta<1$. Let $n \geqslant n_{0}$ be a natural number such that $\lambda_{1}, \ldots, \lambda_{r} \in \sigma_{l e}\left(T^{*}\right) \cap G(n), \tilde{b_{1}^{*}}, \ldots, \tilde{b_{s}^{*}} \in$ $M(n)^{\perp} \mid q X$. Since

$$
D=\left\{\widetilde{q}^{*} f\left(\widetilde{B}_{n}^{*}\right) \tilde{b}_{j}^{*} ; f \in H^{\infty}(G) \text { with }\|f\| \leqslant 1, j=1,2, \ldots, s\right\}
$$

is compact, there are vectors $x_{-t}^{*}, \ldots, x_{-1}^{*}, x_{0}^{*} \in X^{*}$ such that

$$
\min \left\{\left\|x^{*}-x_{-k}^{*}\right\| ; k=0,1, \ldots, t\right\}<\delta
$$


for all $x^{*} \in D$. Define

$$
X_{0}^{*}=\left\{a_{1}, \ldots, a_{s}\right\}^{\perp}
$$

By [10, Lemma III.1.1] there is a closed finite codimensional subspace $X_{1}^{*}$ in $X^{*}$ such that

$$
\alpha\left(\bigvee\left\{x_{-t}^{*}, \ldots, x_{-1}^{*}, x_{0}^{*}\right\}, X_{1}^{*}\right)>1-\delta
$$

Thus by Lemma 6 there are vectors $x_{1}^{*} \in X_{0}^{*} \cap X_{1}^{*}, z_{1}^{*} \in Z^{*}, \widetilde{z_{1}^{* \prime}} \in M(n)^{\perp} \mid q X$, such that

$$
\left\|x_{1}^{*}\right\|=1, \widetilde{q}^{*} \tilde{z_{1}^{*}}=x_{1}^{*},\left\|\left(\lambda_{1}-\widetilde{B}^{*}\right) \tilde{z_{1}^{*}}\right\|<\delta,\left\|\widetilde{z_{1}^{*}}-\widetilde{z_{1}^{* \prime}}\right\|<\delta,
$$

where $\tilde{z_{1}^{*}}=z_{1}^{*} \mid q X$.

Likewise, by [10, Lemma III.1.1] there is a closed finite codimensional subspace $X_{2}^{*}$ in $X^{*}$ such that

$$
\alpha\left(\bigvee\left\{x_{-t}^{*}, \ldots, x_{0}^{*}, x_{1}^{*}\right\}, X_{2}^{*}\right)>1-\delta
$$

Again by Lemma 6 there are vectors $x_{2}^{*} \in X_{0}^{*} \cap X_{1}^{*} \cap X_{2}^{*}, z_{2}^{*} \in Z^{*}, \widetilde{z_{2}^{* \prime}} \in M(n)^{\perp} \mid q X$ such that

$$
\left\|x_{2}^{*}\right\|=1, \widetilde{q}^{*} \tilde{z_{2}^{*}}=x_{2}^{*},\left\|\left(\lambda_{2}-\widetilde{B}^{*}\right) \tilde{z_{2}^{*}}\right\|<\delta,\left\|\widetilde{z_{2}^{*}}-\widetilde{z_{2}^{* \prime}}\right\|<\delta,
$$

where $\tilde{z_{2}^{*}}=z_{2}^{*} \mid q X$.

Continuing in this way, we obtain vectors $x_{1}^{*}, \ldots, x_{r}^{*} \in X_{0}^{*} ; z_{1}^{*}, \ldots, z_{r}^{*} \in$ $Z^{*} ; \widetilde{z_{1}^{* \prime}}, \ldots, \widetilde{z_{r}^{* \prime}} \in M(n)^{\perp} \mid q X$ such that

$$
\begin{gathered}
\alpha\left(\bigvee\left\{x_{-t}^{*}, \ldots, x_{k-1}^{*}\right\}, \bigvee\left\{x_{k}^{*}, \ldots, x_{r}^{*}\right\}\right)>1-\delta, \\
\left\|x_{k}^{*}\right\|=1, \tilde{q}^{*} \tilde{z_{k}^{*}}=x_{k}^{*},\left\|\left(\lambda_{k}-\widetilde{B}^{*}\right) \widetilde{z_{k}^{*}}\right\|<\delta,\left\|\widetilde{z_{k}^{*}}-\widetilde{z_{k}^{* \prime}}\right\|<\delta,
\end{gathered}
$$

where $k=1,2, \ldots, r$, and $\tilde{z_{k}^{*}}=z_{k}^{*} \mid q X$.

An easy calculation shows that

$$
\max \left\{\left|\alpha_{k}\right| ; k=1,2, \ldots, r\right\} \leqslant \frac{2}{1-\delta}\left\|\sum_{k=1}^{r} \alpha_{k} x_{k}^{*}\right\|
$$

holds for all $\alpha_{1}, \ldots, \alpha_{r} \in C$ and that the canonical projection of $L=\bigvee_{k=-t}^{r} x_{k}^{*}$ onto $\bigvee_{k=1}^{r} x_{k}^{*}$ has norm less than $2 /(1-\delta)$. By Zenger's Lemma (see [11] or $\left[3, \begin{array}{c}k=-t \\ \text { p.20]) there }\end{array}\right.$ 
exist a linear functional $\varphi$ on $L$ and complex numbers $\mu_{1}, \ldots, \mu_{r}$ such that $\|\varphi\|<$ $2 /(1-\delta)$ and

$$
\begin{aligned}
\left\|\sum_{k=1}^{r} \mu_{k} x_{k}^{*}\right\| \leqslant 1, \quad \varphi\left(\mu_{k} x_{k}^{*}\right) & =c_{k}(k=1,2, \ldots, r), \\
\varphi\left(x_{k}^{*}\right) & =0(k=0,-1, \ldots,-t) .
\end{aligned}
$$

Using the canonical isometric identification $L^{*}=X /{ }^{\perp} L$ we can choose a vector $x \in X$ such that $\|x\|<2 /(1-\delta)$ and

$$
\left\langle x, x_{k}^{*}\right\rangle=\varphi\left(x_{k}^{*}\right), k=-t, \ldots,-1,0,1, \ldots, r .
$$

If $\delta$ is chosen small enough, then $\|x\|<2 /(1-\delta) \leqslant 3$.

Let $\tilde{z^{*}}=\sum_{k=1}^{r} \tilde{\mu_{k}} \widetilde{z_{k}^{* \prime}}$. It follows from (8.3), (8.4) and (8.5) that if $\delta$ is chosen small enough, then

$$
\left\|\widetilde{q}^{*} \tilde{z}^{*}\right\| \leqslant\left\|\sum_{k=1}^{r} \mu_{k} \widetilde{q^{*}} \widetilde{z_{k}^{*}}\right\|+\left\|\sum_{k=1}^{r} \mu_{k} \widetilde{q}^{*}\left(\widetilde{z_{k}^{* \prime}}-\widetilde{z_{k}^{*}}\right)\right\| \leqslant 1+\left\|\widetilde{q}^{*}\right\| \delta(2 r /(1-\delta)) \leqslant 2 .
$$

It will now be shown that $\left\|x \otimes \tilde{z^{*}}-\sum_{k=1}^{r} c_{k} E_{\lambda_{k}}\right\| \leqslant \varepsilon$. In fact, it follows from (8.3), (8.5) and (8.6) that

$$
c_{k}=\varphi\left(\mu_{k} x_{k}^{*}\right)=\mu_{k}\left\langle x, x_{k}^{*}\right\rangle=\mu_{k}\left\langle x, \widetilde{q}^{*} \tilde{z_{k}^{*}}\right\rangle
$$

for each $k=1,2, \ldots, r$. So by (8.3) if $\delta$ is chosen small enough, then the estimate

$$
\begin{aligned}
\mid x & \otimes \tilde{z}^{*}(f)-\sum_{k=1}^{r} c_{k} E_{\lambda_{k}}(f)|=| \sum_{k=1}^{r} u_{k}\left(\left\langle x, \widetilde{q}^{*} f\left(\widetilde{B}_{n}^{*}\right) \widetilde{z_{k}^{* \prime}}\right\rangle-\left\langle x, \widetilde{q}^{*} f\left(\lambda_{k}\right) \widetilde{z_{k}^{*}}\right\rangle\right) \mid \\
& =\left|\sum_{k=1}^{r} \mu_{k}\left\langle x, \widetilde{q}^{*} f_{\lambda_{k}}\left(\widetilde{B}_{n}^{*}\right)\left(\widetilde{B}^{*}-\lambda_{k}\right) \widetilde{z_{k}^{* \prime}}\right\rangle\right|+\left|\sum_{k=1}^{r} \mu_{k}\left\langle x, \widetilde{q}^{*} f\left(\lambda_{k}\right)\left(\widetilde{z_{k}^{* \prime}}-\widetilde{z_{k}^{*}}\right)\right\rangle\right|<\varepsilon,
\end{aligned}
$$

holds for all $f \in H^{\infty}(G)$ with $\|f\| \leqslant 1$.

Next it will be shown that $\left\|x \otimes \widetilde{b_{j}^{*}}\right\| \leqslant \varepsilon$ for each $j=1,2, \ldots, s$. In fact, it follows from (8.5) and (8.6) that

$$
\left\langle x, x_{-k}^{*}\right\rangle=\varphi\left(x_{-k}^{*}\right)=0
$$

for each $k=0,1, \ldots, t$. So by (8.1) if $\delta$ is chosen small enough, then the estimate

$$
\begin{aligned}
\left|x \otimes \tilde{b_{j}^{*}}(f)\right| & =\min \left\{\left|\left\langle x, \widetilde{q}^{*} f\left(\widetilde{B}_{n}^{*}\right) \tilde{b_{j}^{*}}-x_{-k}^{*}\right\rangle\right| ; k=0,1, \ldots, t\right\} \\
& \leqslant\|x\| \cdot \min \left\{\left\|\widetilde{q}^{*} f\left(\widetilde{B}_{n}^{*}\right) \tilde{b_{j}^{*}}-x_{-k}^{*}\right\|, k=0,1, \ldots, t\right\}<\varepsilon
\end{aligned}
$$


holds for each $j=1,2, \ldots, s$. and all $f \in H^{\infty}(G)$ with $\|f\| \leqslant 1$.

Finally, we show that $\left\|a_{j} \otimes \tilde{z^{*}}\right\| \leqslant \varepsilon$ for each $j=1,2, \ldots, s$. In fact, by (8.2), (8.3) and $x_{k}^{*} \in X_{0}^{*}(k=1,2, \ldots, r)$ we obtain

$$
\left\langle a_{j}, \widetilde{q^{*}} \tilde{z_{k}^{*}}\right\rangle=\left\langle a_{j}, x_{k}^{*}\right\rangle=0
$$

for all $k=1,2, \ldots, r$, and $j=1,2, \ldots, s$. So by (8.3) if $\delta$ is chosen small enough, then the estimate

$$
\begin{aligned}
& \left|a_{j} \otimes \tilde{z}^{*}(f)\right|=\left|\sum_{k=1}^{r} \mu_{k}\left(\left\langle a_{j}, \widetilde{q}^{*} f\left(\widetilde{B}_{n}^{*}\right) \widetilde{z_{k}^{* \prime}}\right\rangle-\left\langle a_{j}, \widetilde{q}^{*} f\left(\lambda_{k}\right) \tilde{z_{k}^{*}}\right\rangle\right)\right| \\
& =\left|\sum_{k=1}^{r} \mu_{k}\left\langle a_{j}, \widetilde{q}^{*} f_{\lambda_{k}}\left(\widetilde{B}_{n}^{*}\right)\left(\widetilde{B}^{*}-\lambda_{k}\right) \widetilde{z_{k}^{* \prime}}\right\rangle\right|+\left|\sum_{k=1}^{r} \mu_{k}\left\langle a_{j}, \widetilde{q}^{*} f\left(\lambda_{k}\right)\left(\widetilde{z_{k}^{* \prime}}-\widetilde{z_{k}^{*}}\right)\right\rangle\right|<\varepsilon
\end{aligned}
$$

holds for each $j=1,2, \ldots, s$ and all $f \in H^{\infty}(G)$ with $\|f\| \leqslant 1$.

LEMMA 9. For any given $\lambda \in G$, there exist sequences $\left\{x_{n}\right\}_{n=0}^{\infty}$ in $X$ and $\left\{\widetilde{z_{n}^{*}}\right\}_{n=0}^{\infty}$ in $M(G)$ such that

(1) $\left\|E_{\lambda}-x_{n} \otimes \widetilde{z_{n}^{*}}\right\|<2^{-2 n+1}, n=0,1,2, \ldots$,

(2) $\left\|x_{n}-x_{n-1}\right\|<2^{-n+5},\left\|\widehat{q}^{*}\left(\widetilde{z_{n}^{*}}-\widetilde{z_{n-1}^{*}}\right)\right\|<2^{-n+5}, n=1,2, \ldots$.

Proof: Let $x_{0}=0 \in X, \tilde{z_{0}^{*}}=0 \in M(G)$. Assume by induction that we have constructed the vectors $x_{0}, x_{1}, \ldots, x_{n-1} \in X ; \widetilde{z_{0}^{*}}, \widetilde{z_{1}^{*}}, \ldots, \widetilde{z_{n-1}^{*}} \in M(G)$ such that

$$
\begin{aligned}
\left\|E_{\lambda}-x_{k} \otimes \tilde{z_{k}^{*}}\right\| & <2^{-2 k+1}, \quad k=1, \ldots, n-1, \\
\left\|x_{k}-x_{k-1}\right\| & <2^{-k+5}, \quad k=1,2, \ldots, n-1, \\
\left\|\tilde{q}^{*}\left(\tilde{z_{k}^{*}}-\widetilde{z_{k-1}^{*}}\right)\right\| & <2^{-k+5}, \quad k=1,2, \ldots, n-1 .
\end{aligned}
$$

We now wish to construct $x_{n} \in X, \widetilde{z_{n}^{*}} \in M(G)$ satisfying (9.1), (9.2) and (9.3) for $k=n$.

By Note $1, \sigma_{l e}\left(T^{*}\right)$ is dominating in $G$. Thus by [6, Proposition 2.8] (or [4, Lemma 4.4]) and (9.1), there are complex numbers $\lambda_{1}, \ldots, \lambda_{r} \in \sigma_{l e}\left(T^{*}\right) \cap G$ and complex numbers $c_{1}, \ldots, c_{r}$ such that

$$
\sum_{j=1}^{r}\left|c_{j}\right| \leqslant 2^{-2 n+3},\left\|E_{\lambda}-x_{n-1} \otimes \widetilde{z_{n-1}^{*}}-\sum_{j=1}^{r} c_{j} E_{\lambda_{j}}\right\|<2^{-2 n-2} .
$$

Write $c_{j}=c_{1 j}-c_{2 j}+i c_{3 j}-i c_{4 j}$, where $j=1,2, \ldots, r, i=\sqrt{-1}$ and $0 \leqslant c_{k_{j}} \leqslant\left|c_{j}\right|$. Then

$$
\sum_{j=1}^{r} c_{k_{j}} \leqslant \sum_{j=1}^{r}\left|c_{j}\right|, k=1,2,3,4 .
$$


Without loss of generality, we assume that for each $k$, the real numbers $c_{k 1}, \ldots, c_{k r}$ are not all zero. Set $c_{k j}^{\prime}=c_{k j} / \sum_{j=1}^{r} c_{k j}$, then $c_{k j}^{\prime} \geqslant 0$ and $\sum_{j=1}^{r} c_{k j}^{\prime}=1$. Consequently, for any given real number $\varepsilon>0$, by Lemma 8 we can choose successively pairs $\left(x^{(k)}, \tilde{z}^{(k)}\right)_{1 \leqslant k \leqslant 4}$ of vectors $x^{(k)} \in X, \tilde{z}^{(k)} \in M(G)$ such that

$$
\begin{aligned}
& \left\|x^{(k)}\right\| \leqslant 3,\left\|\widetilde{q}^{*} \widetilde{z^{(k)}}\right\| \leqslant 2,\left\|x^{(k)} \otimes \widetilde{z^{*}(k)}-\sum_{j=1}^{r} c_{k j}^{\prime} E_{\lambda_{j}}\right\| \leqslant \varepsilon, \\
& \left\|x_{n-1} \otimes \widetilde{z^{*}(k)}\right\| \leqslant \varepsilon,\left\|x^{(k)} \otimes \widetilde{z_{n-1}^{*}}\right\| \leqslant \varepsilon,\left\|x^{(k)} \otimes \widetilde{z^{*}(m)}\right\| \leqslant \varepsilon,
\end{aligned}
$$

for all $k, m=1,2,3,4$ with $k \neq m$. Define

$$
\begin{aligned}
& x_{n}=x_{n-1}+\left(\sum_{j=1}^{r} c_{1 j}\right)^{1 / 2} x^{(1)}-\left(\sum_{j=1}^{r} c_{2 j}\right)^{1 / 2} x^{(2)}+i\left(\sum_{j=1}^{r} c_{3 j}\right)^{1 / 2} x^{(3)}-i\left(\sum_{j=1}^{r} c_{4 j}\right)^{1 / 2} x^{(4)}, \\
& \widetilde{z_{n}^{*}}=\widetilde{z_{n-1}^{*}}+\sum_{k=1}^{4}\left(\sum_{j=1}^{r} c_{k j}\right)^{1 / 2} \widetilde{z^{(j)}} .
\end{aligned}
$$

Then it follows from (9.5) and (9.6) that the estimate

$$
\begin{aligned}
\| x_{n} \otimes & \widetilde{z_{n}^{*}}-x_{n-1} \otimes \widetilde{z_{n-1}^{*}}-\sum_{j=1}^{r} c_{j} E_{\lambda_{j}} \| \\
\leqslant & \left(\sum_{j=1}^{r}\left|c_{j}\right|\right)^{1 / 2}\left(\sum_{k=1}^{4}\left\|x_{n-1} \otimes \widetilde{z^{*}(k)}\right\|+\sum_{k=1}^{4}\left\|x^{(k)} \otimes \widetilde{z_{n-1}^{*}}\right\|\right) \\
& +\sum_{j=1}^{r}\left|c_{j}\right|\left(\sum_{k \neq m}\left\|x^{(k)} \otimes \widetilde{z^{*}(m)}\right\|\right) \\
& +\sum_{j=1}^{r}\left|c_{j}\right|\left(\sum_{k=1}^{4}\left\|x^{(k)} \otimes \widetilde{z^{*}(k)}-\sum_{j=1}^{r} c_{k j}^{\prime} E_{\lambda_{j}}\right\|\right) \\
\leqslant & 8 \varepsilon\left(\sum_{j=1}^{r}\left|c_{j}\right|\right)^{1 / 2}+16 \varepsilon \sum_{j=1}^{r}\left|c_{j}\right|<2^{-2 n-2} .
\end{aligned}
$$

holds, if $\varepsilon$ is chosen small enough. Hence it follows from (9.4), (9.5) and (9.6) that

$$
\begin{aligned}
& \left\|E_{\lambda}-x_{n} \otimes \widetilde{z_{n}^{*}}\right\|<2^{-2 n+1}, \\
& \left\|x_{n}-x_{n-1}\right\| \leqslant 12\left(\sum_{j=1}^{r}\left|c_{j}\right|\right)^{1 / 2}<2^{-n+5}, \\
& \left\|\widetilde{q}\left(\widetilde{z_{n}^{*}}-\widetilde{z_{n-1}^{*}}\right)\right\| \leqslant 8\left(\sum_{j=1}^{r}\left|c_{j}\right|\right)^{1 / 2}<2^{-n+5} .
\end{aligned}
$$


Proof of Theorem 1: Fix an arbitrary point $\lambda \in G$. By Lemma 9, there exist sequences $\left\{x_{n}\right\}_{n=0}^{\infty}$ in $X$ and $\left\{\widetilde{z_{n}^{*}}\right\}_{n=0}^{\infty}$ in $M(G)$ such that

(a) $\left\|E_{\lambda}-x_{n} \otimes \widetilde{z_{n}^{*}}\right\|<2^{-2 n+1}, n=0,1,2, \ldots$

(b) $\left\|x_{n}-x_{n-1}\right\|<2^{-2 n+5},\left\|\widetilde{q}^{*}\left(\widetilde{z_{n}^{*}}-\widetilde{z_{n-1}^{*}}\right)\right\|<2^{-2 n+5}, n=1,2, \ldots$

The inequality in (b) ensures that the sequences $\left\{x_{n}\right\}_{n=0}^{\infty}$ and $\left\{\widetilde{q}^{*} \widetilde{z}_{n}^{*}\right\}_{n=0}^{\infty}$ are Cauchy in $X$ and $X^{*}$, respectively. Hence, there are vectors $x \in X$ and $x^{*} \in X^{*}$ such that

$$
\lim _{n \rightarrow \infty} x_{n}=x, \lim _{n \rightarrow \infty} \widetilde{q^{*}} \widetilde{z_{n}^{*}}=x^{*}
$$

Since $\widetilde{z_{n}^{*}} \in M(G)$, there is a natural number $m(n) \geqslant n_{0}$ such that $\widetilde{z_{n}^{*}} \in M(m(n))^{\perp} \mid q X$. Thus there exists $z_{n}^{*} \in M(m(n))^{\perp}$ such that $\widetilde{z_{n}^{*}}=z_{n}^{*} \mid q X$. Consequently, for any polynomial $p$, by Lemma 2, Lemma 3 and (a) we obtain

$$
\begin{aligned}
p(\lambda) & =E_{\lambda}(p)=\lim _{n \rightarrow \infty} x_{n} \otimes \widetilde{z_{n}^{*}}(p) \\
& =\lim _{n \rightarrow \infty}\left\langle x_{n}, \widetilde{q}^{*} p\left(\widetilde{B}_{m(n)}^{*}\right) \widetilde{z_{n}^{*}}\right\rangle=\lim _{n \rightarrow \infty}\left\langle x_{n}, \widetilde{q}^{*} p\left(\widetilde{B^{*}}\right) \widetilde{z_{n}^{*}}\right\rangle \\
& =\lim _{n \rightarrow \infty}\left\langle x_{n}, q^{*} p\left(B^{*}\right) z_{n}^{*}\right\rangle=\lim _{n \rightarrow \infty}\left\langle x_{n}, p\left(T^{*}\right) q^{*} z_{n}^{*}\right\rangle \\
& =\lim _{n \rightarrow \infty}\left\langle p(T) x_{n}, \widetilde{q^{*}} \widetilde{z_{n}^{*}}\right\rangle=\left\langle p(T) x, x^{*}\right\rangle .
\end{aligned}
$$

Therefore $\left\langle x, x^{*}\right\rangle=1$ and $\left\langle(T-\lambda)^{n} x, x^{*}\right\rangle=0$ for all $n=1,2, \ldots$ Hence $x \neq 0$, $x^{*} \neq 0$, and $(T-\lambda)^{n} x \in \operatorname{ker} x^{*}$ for all $n=1,2, \ldots$ Two cases may arise: $(T-\lambda) x=$ 0 or $(T-\lambda) x \neq 0$. If $(T-\lambda) x=0$, then $\operatorname{ker}(T-\lambda)$ is an invariant subspace of $T$. If $(T-\lambda) x \neq 0$, then we write $L_{0}=\bigvee\left\{(T-\lambda) x,(T-\lambda)^{2} x, \ldots\right\}$, and hence $L_{0} \subset \operatorname{ker} x^{*}$. Consequently, it follows from $x^{*} \neq 0$ that $L_{0} \neq\{0\}$ and $L_{0} \neq X$. It is easy to see that $L_{0}=\bigvee\left\{T x, T^{2} x, \ldots\right\}$ if $\lambda=0$, and $L_{0}=\bigvee\left\{x, T x, T^{2} x, \ldots\right\}$ if $\lambda \neq 0$. Therefore $L_{0}$ is an invariant subspace of $T$.

\section{REFERENCES}

[1] E. Albrecht, and B. Chevreau, 'Invariant subspaces of $l^{p}$-operators having Bishop's property $(\beta)$ on a large part of their spectrum', J. Operator Theory 18 (1987), 339-372.

[2] C. Apostol, 'The spectral flavour of Scott Brown's techniques', J. Operator Theory 6 (1981), 3-12.

[3] F.F. Bonsall and J. Duncan, Numerical manges, II (Cambridge University Press, Cambridge, 1973).

[4] S. Brown, 'Some invariant subspaces for subnormal operators', Integral Equations Operator Theory 1 (1978), 310-330.

[5] S. Brown, 'Hyponormal operators with thick spectra have invariant subspaces', Ann. of Math. 125 (1987), 93-103. 
[6] S. Brown, B. Chevreau and C. Pearcy, 'Contractions with rich spectrum have invariant subspaces', J. Operator Theory 1 (1979), 123-136.

[7] J. Eschmeier, 'Operators with rich invariant subspace lattices', J. Reine. Angew. Math. 396 (1989), 41-69.

[8] J. Eschmeier and B. Prunaru, 'Invariant subspaces for operators with Bishop's property $(\beta)$ and thick spectrum', J. Funct. Anal. 94 (1990), 196-222.

[9] H. Mohebi and M. Radjabalipour, 'Scott Brown's techniques for perturbations of decomposable operators', Integral Equations Operator Theory 18 (1994), 222-241.

[10] I. Singer, Bases in Banach spaces, II (Springer-Verlag, Berlin, Heidelberg, New York, 1981).

[11] C. Zenger, 'On convexity properties of the Bauer field of values of a matrix', Numer. Math. 12 (1968), 96-105.

Department of Mathematics

Fujian Normal University

Fuzhou, Fujian 350007

People's Republic of China

e-mail: liumingx@pub3.fz.fj.cn 